\title{
Fluorescence Bronchoscopy
}

National Cancer Institute

\section{Source}

National Cancer Institute. Fluorescence Bronchoscopy. NCI Thesaurus. Code C20035.

Fluorescence bronchoscopy is based on the fact that normal tissue fluoresces differently than abnormal tissue when exposed to an appropriate waveleng th of light and that this difference can be detected. 